\title{
In search of health evidence. Proposal for self-learning
}

\author{
Blasco-Igual, Jose María ${ }^{\text {; }}$ Sanchis-Sánchez, Enriquea; García-Molina, Pablo ; \\ Balaguer-López, Evelyn ${ }^{b}$ Georgieva, Sylvia ${ }^{c}$ and Sánchez-Lorente, María \\ Montserrat $^{\mathrm{d}}$
}

${ }^{\mathrm{a}}$ Department of Physiotherapy, University of Valencia, Spain. ${ }^{\mathrm{b}}$ Department of Nursing, University of Valencia, Spain. ' Department of Methodology and Behavioral Sciences, University of Valencia, Spain and ${ }^{\mathrm{d}}$ Conselleria de Sanitat, Generalitat Valenciana. Spain.

\begin{abstract}
Information sources are a key tool in the current social and technological evolution. While it is true that not all are benefits. In the same way the over information is evident, and can lead to the extraction of conclusions that, in many cases, becomes confusing, diffuse, or even erroneous. In this context, the use of the 65 pyramid can help you find the best research evidence with the least amount of time and effort. This work proposal exposes the methods and development of a support and training program to search for evidence in health. To do so, the learning resources will be taught through a specific seminar and the option of using self-directed online resources, freely accessible, from the "National Collaborating Center for Methods and Tools" (NCCMT) of McMaster University. Later, the assessment of competences will be undertaken. The expected results for the students is to be able to perform the analysis of their searches based on elements that can later include in their final work, with the benefits that this implies in the education.
\end{abstract}

Keywords: $\quad$ Self-learning; $\quad$ Quality-Assessed; $\quad$ Evidence-Informed; Preappraised Resources; 6S Pyramid. 


\section{Introduction}

Information sources are a key tool in the current social and technological evolution. However, it is true that not all are benefits. In the same way the over information is evident, and can lead to the extraction of conclusions that, in many cases, becomes confusing, diffuse, or even erroneous. Therefore, it is necessary to know how to discern and contrast the evidence that students use (in many cases) indiscriminately to complete the large number of jobs that new teaching methodologies and education plans require for the attainment of competences.

In past years, accessing the evidence meant having knowledge of literature search and the ability to apply of critical appraisal skills to separate lower-from higher-quality clinical studies. The databases and other sources that should be searched to identify evidence of clinical effectiveness depend on the review question (National Institute for Health and Care Excellence, 2012). However, over the last decade, many practical resources have been created to facilitate ready access to high-quality research. Currently, new sources of information are available to help us recover the best updated evidence quickly. These are called "Preappraised Resources" because they are the result of a filtering process and only include high quality studies and also because they are updated regularly (DiCenso, Bayley, \& Haynes, 2009).

In 2001, R. Brian Haynes (one of the natural leaders of the Evidence-Based Medicine Working Group) synthesized, in a pyramidal model of four strata, the information resources according to their usefulness and properties in the decision making in health care. This hierarchical structure was called the "4S" pyramid, by the initials in English of the four resources that comprise it: Systems, Synopses, Syntheses and Studies. The same author added, in 2006, one stratum more to the pyramid (Summaries), known, therefore, as the pyramid of the «5S». Finally, in 2011 the Synopses were divided into two groups (Synopses of Studies and Synopses of Syntheses). In this final pyramid of the «6S», the ascending levels involve a smaller volume of information, but a greater degree of processing of it.

The use of the $6 \mathrm{~S}$ pyramid can help you find the best research evidence with the least amount of time and effort.

Each level of the 6S pyramid (Figure 1) is based on research evidence from the lower layers, so starting a search at the top (or the highest possible layer of the $6 \mathrm{~S}$ pyramid model) yields the most synthesized research evidence and of the highest quality (DiCenso et al., 2009). 


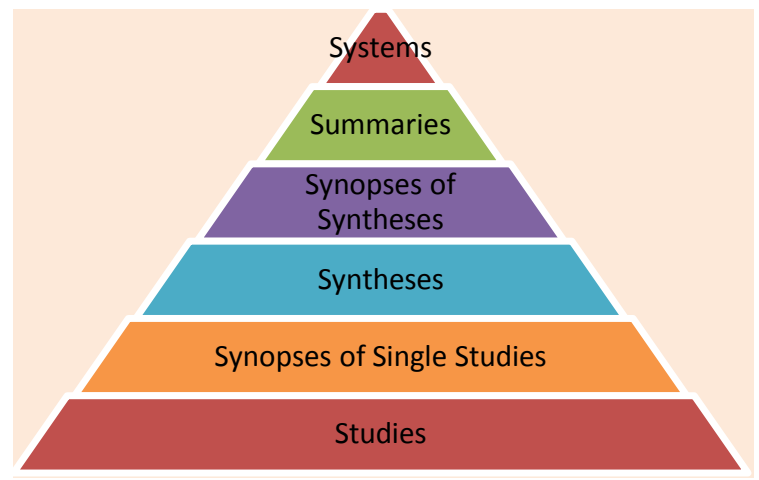

Figure 1. 6S pyramid.

Most health sciences students initiate the search by the lowest level, this means that the original studies are used. However, it is necessary to understand how to use the search engines to find the study that interests and then finally to interpret and apply the individual study without an expert opinion. This search strategy as well as being a tedious and slow process does not guarantee that the best available information is actually used.

It is essential to modify this erroneous practice progressively. For all these reasons, this educational innovation activity is suggested with two objectives; to know the use of different sources of information or resources according to the needs of each research question and to classify the different types of source and resources according to the Haynes pyramid.

Evidence-based practice is an integral component of the health professionals' curriculum in undergraduate, postgraduate and clinical practice training. The concepts of levels of evidence and degrees of recommendations are fundamental for the definition of evidencebased practice, as they attempt to standardize and provide professionals with convincing rules to evaluate published research, determine its validity and summarize its usefulness in clinical practice (Upshur, 2003).

McMaster University is one of the institutions that leads internationally the harmonization of evidence hierarchies and the understanding of what is an evidence-based recommendation. This understanding allows for more consistent patterns of practice in order to benefit the patient.

The central focus of open learning is commonly placed on the "needs of the student as perceived by the student" learning as an innovation both within and across academic disciplines, institutions of higher education, collaborative initiatives between institutions and education for young learners (D’Antoni, 2009). 
Generally, through open learning, activities that enhance learning opportunities within formal education systems or expand learning opportunities beyond formal education systems are carried out. It is not limited to classroom teaching methods or learning interactive approaches (D’Antoni, 2009).

\section{Proposal}

All undergraduate students have to perform works in which they must know and assess the scientific evidence of the subject on which they have to carry out the required activity in the teaching guides of the different subjects

In order to perform an effective literature search, students have to know how to clearly define the question or problem to be studied and to know which is the most appropriate research design to answer the question asked. The next issue that students have to deal with is the one that is undertaken in this activity: knowing where to look for information with the best available evidence to address the problem and also to establish a search strategy that allows to find the most solid quality and most relevant evidence.

Development of a support and training program to search for evidence in health, based on learning resources through a specific seminar and the option of using self-directed online resources, freely accessible, from the "National Collaborating Center for Methods and Tools" (NCCMT) of McMaster University.

These online learning resources have been developed to support the process of evidenceinformed public health. Each module relates to at least one step in the process as indicated by Dobbins (Dobbins, 2017): 1) define, 2) search, 3) appraise, 4) synthsize, 5) adapt, 6)

implement, 7) evaluate. Our proposal is related to the search module. Registration is free and simple.

\subsection{Aims}

The proposal has the following objectives:

- To know the use of different sources of information or resources according to the needs of each clinical question.

- To classify the different types of sources and resources according to the Haynes pyramid. 


\subsection{Methodology}

The planned methodology contains the following steps: To groups

A. Conducting an initial training seminar, which will consist of two parts.

1. Theoretical-practical session in which the "Search for evidence" program will be exposed, in which the evidence will be defined and how to search and discern the level of evidence. The objectives will also be exposed, as well as the sequencing, methodology, and knowledge to be achieved.

A document will be provided as a guide or roadmap that will guide students on the following steps in order to achieve the aims. One hour is expected.

2. Presentation, for those students who choose to complete knowledge and skills, online tools and resources and access to the learning center NCCMT Learning Center to perform the module "Searching for Research Evidence in Public Health".

- The module can be completed at home or on the computers of the faculty.

- Term of completion: Period of achievement of the Program.

- Duration of online training: 3-4 hours.

- Explanation about obtaining certification of competencies of the NCCMT of the McMaster University of those students who complete the module and achieve at least $75 \%$ in the final test.

- Requirements for the use of the tool and resource: average comprehension level of English or French.

- Other features: you can work on the modules individually or as part of a group. Each participant in a group must have an individual NCCMT account. You can choose to complete the NCCMT learning module as an individual or as part of a group.

B. Evaluation of the experience session:

1. Evaluation of the knowledge acquired.

2. Assessment of the difficulties encountered and the degree of satisfaction.

A self-administered questionnaire based on questions with answers with a Likert scale will be used. 
The evaluation will be subjective on the part of the students, and objective on the part of the professors, who will base their criterion not only on the perception but on the evaluation of the papers presented in the subjects.

\subsection{Results}

A total of 58 students from the Faculty of Nursing (2 year) participated, of which 47 were women and 11 were men, with mean age of 20.2 (2.6).

Fifty participants completed the training and the forms that were fulfil before and after the training seminar. The participants shown a slight but significant increase in the knowledge right after the training sessions with $\mathrm{p}<0.05$, as shown in Table 1.

Table 1. Results on knowledge evaluation right after the training

\begin{tabular}{|l|r|r|r|}
\hline & Pre & Post & P-Value \\
\hline $\mathbf{n}$ & 58 & 50 & 0.096 \\
\hline Fails & $6,07(1,98)$ & $5,29(1,67)$ & 0.093 \\
\hline Range & {$[2-11]$} & {$[3-10]$} & \\
\hline Score & $6,21(1.14)$ & $6,69(1,05)$ & \\
\hline
\end{tabular}

The level of satisfaction as appraised in terms of utility, method, organization and teaching team was good with a mean value of 7.83 (1.41) as shown in Table 2.

\begin{tabular}{|c|c|c|c|c|c|}
\hline & Utility & Method & $\begin{array}{c}\text { Organization } \\
\text { and } \\
\text { Resources }\end{array}$ & $\begin{array}{c}\text { Teaching } \\
\text { team }\end{array}$ & $\begin{array}{c}\text { Overall } \\
\text { assessment }\end{array}$ \\
\hline Mean & 7.59 & 7.45 & 7.99 & 8.58 & 7.56 \\
\hline Std & 1.43 & 1.31 & 1.28 & 1.11 & 1.61 \\
\hline Min & 3.00 & 4.50 & 4.50 & 5.00 & 2.00 \\
\hline Max & 10.00 & 10.00 & 10.00 & 10.00 & 10.00 \\
\hline
\end{tabular}

Results in the use of resources used in the group works performed during the course and complying with higher levels of evidence will be available by the end of May 2018 .

\subsection{Conclusion}

These types of activities are of interest because they motivate students and provide a continuous added value that allow students to acquire and strengthen competencies for their future reality in the workplace. 


\section{References}

D'Antoni, S. (2009). Open Educational Resources: reviewing initiatives and issues. Open Learning: The Journal of Open and Distance Learning, 24(1), 3-10. http://doi.org/10.1080/02680510802625443

DiCenso, A., Bayley, L., \& Haynes, R. (2009). ACP Journal Club. Editorial: Accessing preappraised evidence: fine-tuning the $5 \mathrm{~S}$ model into a $6 \mathrm{~S}$ model. Ann Intern Med, 151(6).

Dobbins, M. (2017). Rapid Review Guidebook Steps for conducting a rapid review. Retrieved from:

http://www.nccmt.ca/uploads/media/media/0001/01/a816af720e4d587e13da6bb307df8c90 7a5dff9a.pdf

National Institute for Health and Care Excellence. (2012). The guidelines manual: Process and methods [PMG6]. Retrieved from:

https://www.nice.org.uk/guidance/pmg6/resources/the-guidelines-manual-pdf2007970804933\%0Ahttps://www.nice.org.uk/process/pmg6/chapter/reviewing-theevidence

Upshur, R. E. G. (2003). Are all evidence-based practices alike? Problems in the ranking of evidence, 169(7), 672-673. 